\title{
GENUS TWO CURVES WITH QUATERNIONIC MULTIPLICATION AND MODULAR JACOBIAN
}

\author{
JOSEP GONZÁLEZ AND JORDI GUÀRDIA
}

\begin{abstract}
We describe a method to determine all the isomorphism classes of principal polarizations of the modular abelian surfaces $A_{f}$ with quaternionic multiplication attached to a normalized newform $f$ without complex multiplication. We include an example of $A_{f}$ with quaternionic multiplication for which we find numerically a curve $C$ whose Jacobian is $A_{f}$ up to numerical approximation, and we prove that it has quaternionic multiplication and is isogenous to $A_{f}$.
\end{abstract}

\section{INTRODUCTION}

During the last years, abelian surfaces have emerged in arithmetic geometry in different contexts, illustrating interesting phenomena and providing examples for practical applications. While the general theory of abelian surfaces is well known, explicit methods are quite recent and not completely developed. For an irreducible abelian surface over a number field, the problem of determining explicitly all the genus two curves whose Jacobian is isomorphic to the given surface is not solved in general.

We began the study of this problem for irreducible principally polarized abelian surfaces in GJGG02. Afterwards, in GGR05 we developed the theoretical results related to the problem for irreducible polarized abelian surfaces, considering specifically nonprincipal polarizations. In both cases, we applied our ideas to modular abelian surfaces, since the apparition of new algorithms for the description of the Jacobians of modular curves makes possible the generation of explicit examples. Unfortunately, the numerical nature of these algorithms leads only numerically tested examples.

We now consider the interesting case of abelian surfaces with quaternionic multiplication, whose rich endomorphism algebra allows the combined application of further well-known techniques. After describing these techniques, we provide an example of a modular abelian surface presented as the Jacobian of a hyperelliptic curve for which, although numerically determined, we can determine its endomorphism ring and prove that it is isogenous to the given surface.

Received by the editor July 10, 2007.

2000 Mathematics Subject Classification. Primary 11G10, 11 G18.

Key words and phrases. Genus two curves, quaternionic multiplication, modular abelian surfaces.

The authors were partially supported by MTM2006-15038-C02-02.

(C)2008 American Mathematical Society Reverts to public domain 28 years from publication 


\section{General facts}

Let $f=\sum_{n>0} a_{n} q^{n}$ be a normalized newform of $S_{2}\left(\Gamma_{0}(N)\right)$ without complex multiplication such that the abelian variety $A_{f} / \mathbb{Q}$ attached by Shimura in Shi71] has dimension 2. The $\mathbb{Q}$-algebra $\operatorname{End}^{0}\left(A_{f}\right):=\operatorname{End}_{\overline{\mathbb{Q}}}\left(A_{f}\right) \otimes \mathbb{Q}$ can only be the real quadratic field generated by the Fourier coefficients $a_{n}$, the matrix algebra $\mathrm{M}_{2}(\mathbb{Q})$ or an indefinite quaternion algebra $\left(\frac{a, b}{\mathbb{Q}}\right)$. We restrict ourselves to the most interesting case that $A_{f}$ has quaternionic multiplication. Indeed, we will see that if there is a principal polarization not defined over $\mathbb{Q}, A_{f}$ can be presented as the Jacobian of a genus two-curve $C$ defined over an imaginary quadratic field which does not admit descent over $\mathbb{Q}$. Then, there are two possibilities: either $C$ is isomorphic to its complex conjugate $\bar{C}$ and, thus, with rational Igusa invariants or the isomorphism classes of the canonical principal polarizations of both curves are different.

We denote by $E=\mathbb{Q}(\sqrt{m})$ the real quadratic number field generated by $\left\{a_{n}, n>0\right\}$, where $m$ is a square-free integer. It is well known that every Hecke operator provides an endomorphism of $A_{f}$ for which $f$ is an eigenvector and that we identify with the eigenvalue of $f$. So we have a natural injection $\mathbb{Z}\left[\left\{a_{n}\right\}\right] \hookrightarrow$ $\operatorname{End}_{\mathbb{Q}}\left(A_{f}\right) \subset \operatorname{End}_{\mathbb{Q}}^{0}\left(A_{f}\right) \simeq E$ which lets us interpret the coefficients $a_{n}$ as endomorphisms of $A_{f}$.

An inner twist of $f$ is a Dirichlet character $\chi$ of conductor dividing $N$ such that ${ }^{\sigma} f=f \otimes \chi$ for some nontrivial $\sigma \in \operatorname{Gal}(\overline{\mathbb{Q}} / \mathbb{Q})$, i.e. $a_{p}=\chi(p)^{\sigma} a_{p}$ for all primes $p \nmid N$. Since $f$ has trivial Nebentypus, $\chi$ must be quadratic (cf. Rib80]); we will write $K=\overline{\mathbb{Q}}^{\text {ker } \chi}$. By Proposition 8 of Shi73 there is $u_{\chi} \in \operatorname{End}_{K}^{0}\left(A_{f}\right)$ sending $f$ and ${ }^{\sigma} f$ to $g(\chi)^{\sigma} f$ and $g(\chi) f$, respectively, where $g(\chi)$ denotes the Gauss sum relative to the conductor of $\chi$. Of course, $u_{\chi}^{2}=\operatorname{disc} K$. From [Rib80, we have the following characterization.

Proposition 2.1. The abelian surface $A_{f}$ has quaternionic multiplication if and only if there is an inner twist $\chi$ such that its conductor is not a norm of the number field $E$. In this case, the quadratic number field $K=\mathbb{Q}(\sqrt{\delta})=\overline{\mathbb{Q}}^{\text {ker } \chi}$ is imaginary and $\operatorname{End}^{0}\left(A_{f}\right)=\operatorname{End}_{K}^{0}\left(A_{f}\right)=\mathbb{Q}\left(u_{\chi}, T_{p}\right) \simeq\left(\frac{\delta, m}{\mathbb{Q}}\right)$, where $T_{p}$ is the Hecke operator at a prime $p$ such that $a_{p} \notin \mathbb{Z}$.

Let $k$ be a subfield of a fixed algebraic closure $\overline{\mathbb{Q}}$ of $\mathbb{Q}$, let $G_{k}$ be the absolute Galois group $\operatorname{Gal}(\overline{\mathbb{Q}} / k)$ and let $A$ be an abelian variety defined over $k$. The class of an invertible sheaf $\mathcal{L}$ (not necessarily defined over $k$ ) in the Néron-Severi group $\operatorname{NS}\left(A_{\overline{\mathbb{Q}}}\right)$ is defined over $k$ if it is invariant by $G_{k}$. Every invertible sheaf $\mathcal{L} \in$ $\operatorname{NS}\left(A_{\overline{\mathbb{Q}}}\right)^{G_{k}}$ defines a morphism $\varphi_{\mathcal{L}}: A \rightarrow \hat{A}$ over $k$ given by $\varphi_{\mathcal{L}}(P)=\tau_{P}^{*}(\mathcal{L}) \otimes \mathcal{L}^{-1}$, where $\tau_{P}$ denotes the translation by $P$. This morphism is an isogeny if and only if $\mathcal{L}$ is nondegenerate. A polarization on $A$ defined over $k$ is the class of algebraic equivalence of an ample invertible sheaf $\mathcal{L} \in \mathrm{NS}\left(A_{\overline{\mathbb{Q}}}\right)^{G_{k}}$. Equivalently, a polarization on $A$ over $k$ is an isogeny $\lambda: A \rightarrow \hat{A}$ defined over $k$ such that $\lambda \otimes \bar{k}=\varphi_{\mathcal{L}}$ for some ample line bundle $\mathcal{L}$ on $A_{\overline{\mathbb{Q}}}$. The polarization $\mathcal{L}$ is called principal when the degree of $\varphi_{\mathcal{L}}$ is 1.

We proceed to present the results that we will use for our computations.

Proposition 2.2 (cf. Wei57). Let $A / k$ be an abelian surface with a principal polarization $\mathcal{L}$ defined over $k$. If $A$ is simple over $\overline{\mathbb{Q}}$, then $(A, \mathcal{L}) \stackrel{k}{\simeq}\left(\operatorname{Jac}(C), \mathcal{L}\left(\Theta_{C}\right)\right)$, 
where $C / k$ is a smooth curve of genus two and $\mathcal{L}\left(\Theta_{C}\right)$ denotes the canonical principal polarization for $\operatorname{Jac}(C)$.

A nondegenerate invertible sheaf $\mathcal{L}$ on $A$ defined over $k$ induces an anti-involution on the algebra of endomorphisms

$$
*: \mathbb{Q} \otimes \operatorname{End}_{k} A \stackrel{\sim}{\rightarrow} \mathbb{Q} \otimes \operatorname{End}_{k} A, \quad t \mapsto \varphi_{\mathcal{L}}^{-1} \cdot \hat{t} \cdot \varphi_{\mathcal{L}} .
$$

Let $\operatorname{End}_{k}^{s} A=\left\{\beta \in \operatorname{End}_{k} A, \beta^{*}=\beta\right\}$ denote the subgroup of symmetric endomorphisms and let $\operatorname{End}_{k+}^{s} A$ be the set of positive symmetric endomorphisms of $A$.

Theorem 2.1 (Proposition 2.1 and Theorem 2.3 of GGR05]). Let $A / k$ be an abelian variety and let $\mathcal{L} \in \mathrm{NS}\left(A_{\overline{\mathbb{Q}}}\right)^{G_{k}}$ be nondegenerate. Then:

(i) For any endomorphism $t \in \operatorname{End}_{k}^{s} A$, there exists a unique $\mathcal{L}^{(t)} \in \operatorname{NS}\left(A_{\overline{\mathbb{Q}}}\right)^{G_{k}}$ such that $\varphi_{\mathcal{L}^{(t)}}=\varphi_{\mathcal{L}} \cdot t$. More precisely, if $E$ and $E_{t}$ denote the alternating Riemann forms attached to $\mathcal{L}$ and $\mathcal{L}^{(t)}$, respectively. Then

$$
E_{t}(x, y)=E(x, t y)=E(t x, y) .
$$

Moreover, if $t$ is a totally positive element, then $\mathcal{L}$ is a polarization if and only if $\mathcal{L}^{(t)}$ is.

(ii) Assume $A$ is principally polarized over $k$. For any choice of a principal polarization $\mathcal{L}_{0}$ on $A$ defined over $k$, there is an isomorphism of groups

$$
\begin{aligned}
\epsilon: \operatorname{NS}\left(A_{\overline{\mathbb{Q}}}\right)^{G_{k}} & \stackrel{\sim}{\rightarrow} \operatorname{End}_{k}^{s} A \\
\mathcal{L} & \mapsto \varphi_{\mathcal{L}_{0}}^{-1} \cdot \varphi_{\mathcal{L}}
\end{aligned}
$$

such that $\mathcal{L} \in \mathrm{NS}\left(A_{\overline{\mathbb{Q}}}\right)^{G_{k}}$ is a polarization if and only if $\epsilon(\mathcal{L}) \in \operatorname{End}_{k+}^{s} A$ and it is principal if and only if $\epsilon(\mathcal{L}) \in \mathrm{Aut}_{k+}^{s} A$. Moreover, $\epsilon^{-1}(t)=\mathcal{L}_{0}^{(t)}$ and the set of all $k$-isomorphism classes of principal polarizations on $A$ defined over $k$ is the set $\epsilon^{-1}\left(\right.$ Aut $\left._{k+}^{s} A / \sim\right)$, where $\beta_{1} \sim \beta_{2}$ means that $\beta_{1}=\beta^{*} \beta_{2} \beta$ for some $\beta \in \operatorname{Aut}_{k} A$.

(iii) Let $\mathcal{L}$ be a polarization on $A$ over $k$ of degree $d \geq 1$. Then, $A$ is principally polarizable over a number field $k$ if and only if there exists $\gamma \in \operatorname{End}_{k+}^{s}(A)$ satisfying $\operatorname{deg} \gamma=d^{2}$ and $\mathcal{L}^{\left(\gamma^{-1}\right)} \in \operatorname{NS}\left(A_{k}\right)^{G_{k}}$.

Proof. Part (i) and part (ii) can be found in Proposition 2.1 and Theorem 2.3 of GGR05, respectively. Part (iii) follows from part (ii) by using the same arguments as in Corollary 2.12 of GGR05] and taking into account that $\operatorname{deg} \mathcal{L}=d$ is equivalent to $\operatorname{deg} \varphi_{\mathcal{L}}=d^{2}$.

In the particular case that $A$ is a surface and $\operatorname{End}^{0}(A)$ is an indefinite quaternion algebra, we have the following results.

Theorem 2.2 (Theorem 1.1 of $\operatorname{Rot03}$ ). Assume that $\operatorname{End}(A)$ is a maximal order of an indefinite quaternion algebra of discriminant $D$. Then, $A$ is principally polarized and the number $\pi(A)$ of isomorphism classes of principal polarizations of $A$, is

$$
\pi(A)= \begin{cases}\frac{h(-4 D)+h(-D)}{2} & \text { if } D \equiv-1 \quad(\bmod 4), \\ \frac{h(-4 D)}{2} & \text { otherwise. }\end{cases}
$$


Proposition 2.3 (Lemma 4.3 of [BFGR06]). Let $A / \mathbb{Q}$ be an abelian surface such that $\operatorname{End}_{\mathbb{Q}}^{0}(A)$ is the real quadratic number field $\mathbb{Q}(\sqrt{m})$ and $\operatorname{End}(A)$ is a maximal order in a quaternion algebra of discriminant $D$. Then, $A$ admits a polarization of degree $d>0$ defined over $\mathbb{Q}$ if and only if $\operatorname{End}_{k}^{0}(A) \simeq\left(\frac{-D d, m}{\mathbb{Q}}\right)$. In particular, $A$ is the jacobian of a genus 2 curve defined over $\mathbb{Q}$ if and only if the algebra $\left(\frac{-D, m}{\mathbb{Q}}\right)$ ramifies exactly at the primes dividing $D$.

\section{Determination of Curves with Jacobian isomorphic to $A_{f}$}

Let us write $E=\mathbb{Q}(\sqrt{m}), K=\mathbb{Q}(\sqrt{\delta})$ with $m>0, \delta<0$ square-free integers. We denote by $i \in \operatorname{End}_{\mathbb{Q}}^{0}\left(A_{f}\right)$ the (fractional) Hecke operator such that $i^{2}=m$. We take $j \in \operatorname{End}_{K}^{0}\left(A_{f}\right)$ as $u_{\chi}$ if the discriminant of $K$ is $\equiv 1(\bmod 4)$ and as or $u_{\chi} / 4$ otherwise. Then $j^{2}=\delta$ and we set $k:=i \cdot j=-j \cdot i$. Let us denote $\mathcal{O}$ the order $\operatorname{End}\left(A_{f}\right)$.

In order to apply Theorem 2.1, we have to determine first the order $\mathcal{O}$ in $\mathbb{Q}(i, j)$. Once we know $\operatorname{End}^{s}\left(A_{f}\right)$ and $\operatorname{End}_{+}^{s}\left(A_{f}\right)$, we are able to compute the number of isomorphism classes of principal polarizations and we can determine hyperelliptic equations for each one of these polarizations by applying the procedure described in GJGG02.

3.1. The ring of endomorphims of $A_{f}$. Let $A / k$ be an abelian variety of dimension $n$ over a subfield $k$ of $\mathbb{C},\left\{\omega_{1}, \cdots, \omega_{n}\right\}$ and let $\left\{c_{1}, \cdots, c_{2 n}\right\}$ be arbitrary bases of $H^{0}\left(A, \Omega_{A / k}^{1}\right)$ and of $H_{1}(A, \mathbb{Z})$, respectively. We can take as period lattice for $A$ :

$$
\Lambda=\left\{\left(\int_{c} \omega_{1}, \cdots, \int_{c} \omega_{n}\right) \mid c \in H_{1}(A, \mathbb{Z})\right\}=\left\langle\left(\int_{c_{i}} \omega_{1}, \cdots, \int_{c_{i}} \omega_{n}\right), i \leq 2 n\right\rangle .
$$

The period matrix $\Omega$ of $A$ with respect to these bases $\omega_{1}, \cdots, \omega_{n}$ and $c_{1}, \cdots, c_{2 n}$ is

$$
\Omega=\left(\int_{c_{i}} \omega_{j}\right)_{1 \leq i \leq 2 n, 1 \leq j \leq n}=\left(\begin{array}{ccc}
\int_{c_{1}} \omega_{1} & \cdots & \int_{c_{2 n}} \omega_{1} \\
\vdots & \vdots & \vdots \\
\int_{c_{1}} \omega_{n} & \cdots & \int_{c_{2 n}} \omega_{n}
\end{array}\right) .
$$

An endomorphism $\phi: A \rightarrow A$ defined over $k$ induces an endomorphism $\phi^{*}$ in the $k$-vector space $H^{0}\left(A, \Omega_{A / k}^{1}\right)$ and an endomorphism $\phi_{*}$ in the $\mathbb{Z}$-module $H_{1}(A, \mathbb{Z})$. The action of both endomorphisms is related by the equality

$$
\int_{\phi_{*}(c)} \omega=\int_{c} \phi^{*}(\omega) \quad \text { for all } \omega \in H^{0}\left(A, \Omega^{1}\right) \quad \text { and } \quad c \in H_{1}(A, \mathbb{Z}) .
$$

Let us denote by $T \in \mathrm{M}_{n}(k)$ and $M \in \mathrm{M}_{2 n}(\mathbb{Z})$ the matrices of $\phi^{*}$ and $\phi_{*}$ with respect to the chosen bases. It follows from (11) that

$$
{ }^{t} T \cdot \Omega=\Omega \cdot M
$$

Therefore,

$$
M=\left(\begin{array}{c}
\operatorname{Re} \Omega \\
\operatorname{Im} \Omega
\end{array}\right)^{-1} \cdot\left(\begin{array}{c}
\operatorname{Re}\left({ }^{t} T \cdot \Omega\right) \\
\operatorname{Im}\left({ }^{t} T \cdot \Omega\right)
\end{array}\right) .
$$


A $\mathbb{C}$-linear endomorphism $\psi$ on $H^{0}\left(A, \Omega^{1}\right)$ comes from some $\phi \in \mathbb{Q} \otimes \operatorname{End}(A)$, i.e., $\psi=\phi^{*}$, if and only if $\psi(\Lambda)$ is contained in $\mathbb{Q} \otimes \Lambda$, where $\psi(\Lambda)$ is

$$
\begin{aligned}
\left\{\left(\int_{c} \psi\left(\omega_{1}\right), \cdots,\right.\right. & \left.\left.\int_{c} \psi\left(\omega_{n}\right)\right) \mid c \in H_{1}(A, \mathbb{Z})\right\} \\
& =\left\langle\left(\int_{c_{i}} \psi\left(\omega_{1}\right), \cdots, \int_{c_{i}} \psi\left(\omega_{n}\right)\right), 1 \leq i \leq 2 n\right\rangle .
\end{aligned}
$$

As an immediate consequence of equalities (2) and (3), we obtain the next criterion.

Lemma 3.1. If $T$ is the matrix of a $\mathbb{C}$-linear endomorphism $\psi$ of $H^{0}\left(A, \Omega^{1}\right)$ with respect to the basis $\left\{\omega_{1}, \cdots, \omega_{n}\right\}$, then $\psi=\phi^{*}$ for some $\phi \in \mathbb{Q} \otimes \operatorname{End}(A)$ if and only if the matrix

$$
M_{T}:=\left(\begin{array}{c}
\operatorname{Re} \Omega \\
\operatorname{Im} \Omega
\end{array}\right)^{-1} \cdot\left(\begin{array}{c}
\operatorname{Re}\left({ }^{t} T \cdot \Omega\right) \\
\operatorname{Im}\left({ }^{t} T \cdot \Omega\right)
\end{array}\right)
$$

lies in $\mathrm{M}_{2 n}(\mathbb{Q})$ and, moreover,

$$
{ }^{t} T . \Omega=\Omega \cdot M_{T} .
$$

When this is the case, $\phi \in \operatorname{End}(A)$ if and only if $M_{T} \in \mathrm{M}_{2 n}(\mathbb{Z})$.

In our case $A=A_{f}$, Proposition 2.1 gives an explicit description of the action of $\operatorname{End}^{0}\left(A_{f}\right)=\mathbb{Q}(i, j)$ on $H^{0}\left(A_{f}, \Omega^{1}\right)$. Then, to determine the order $\mathcal{O}$ in $\mathbb{Q}(i, j)$ we will only use fractional endomorphisms and, thus, we will only need to check the condition $M_{T} \in \mathrm{M}_{2 n}(\mathbb{Z})$ for a finite number of matrices $T \in \mathrm{M}_{n}(K)$.

3.2. Principal polarizations. We chose a basis $f_{1}, f_{2}$ of the vector space $\left\langle f^{\sigma}, f\right\rangle$ with rational Fourier coefficients. We take $\omega_{i}=f_{i}(q) d q / q, i \leq 2$, as a basis of $H^{0}\left(A_{f}, \Omega_{A_{f} / \mathbb{Q}}^{1}\right)$. We compute with Magma ([BCP97] $)$ the data necessary to describe the subvariety $A_{f}$ : the modular symbols giving a basis of paths for the homology, the period matrix of $\omega_{1}, \omega_{2}$ and the matrix $M_{E}$ of the alternating Riemann form $E$ (with respect to this basis) attached to the canonical polarization $\mathcal{L}$ of the Jacobian of $X_{0}(N)$.

Since $\mathcal{L}$ is defined over $\mathbb{Q}$, the Rosati involution $*$ attached to $\mathcal{L}$ acts on $\operatorname{End}_{\mathbb{Q}}^{0}\left(A_{f}\right)$ as the complex conjugation and hence

$$
i^{*}=i, \quad\left(j^{*}\right)^{2}=\delta, \quad i \cdot j^{*}=-j^{*} \cdot i, \quad k^{*}=j^{*} \cdot i^{*} .
$$

Therefore, $j^{*}= \pm j$ and $k^{*}=\mp k$ and $\operatorname{End}^{s}\left(A_{f}\right)$ is a free $\mathbb{Z}$-module of rank 3 . The action of $*$ on $\operatorname{End}^{0}\left(A_{f}\right)$ viewed as a subfield of $\mathrm{M}_{4}(\mathbb{Q}) \simeq \operatorname{End}\left(\mathbb{Q} \otimes H_{1}\left(A_{f}, \mathbb{Z}\right)\right)$ is the following:

$$
M \mapsto\left(M_{E} \cdot M \cdot M_{E}^{-1}\right)^{t} .
$$

So, once we have determined a $\mathbb{Z}$-basis of the order $\mathcal{O}$, we have the corresponding matrices $M_{i} \in \mathrm{M}_{4}(\mathbb{Z})$ acting on $H^{1}\left(A_{f}, \mathbb{Z}\right)$ with respect to the basis chosen for this $\mathbb{Z}$-module. Then $\operatorname{End}^{s}\left(A_{f}\right)$ is given by the matrices

$$
\left\{M \in \mathbb{Z} M_{1} \oplus \cdots \oplus \mathbb{Z} M_{4}: M^{t} \cdot M_{E}=M_{E} \cdot M\right\},
$$

and $\operatorname{End}_{+}^{s}\left(A_{f}\right)$ is the subset corresponding to the matrices $M$ with positive eigenvalues.

When the polarization $\mathcal{L}$ is of type $(1, d)$ with $d>1$, using part (iii) of Theorem 2.1 we can check whether $A_{f}$ admits principal polarizations and compute all isomorphism classes of them. 
3.3. Rational equations. Once we know that an irreducible abelian surface $A_{f}$ is principally polarizable, we can realize it as the Jacobian variety of a hyperelliptic curve over $K$. We find a $K$-rational model $Y^{2}=F(X)$ of this curve following the procedure described in GJGG02, with two slight corrections. First, the computation of the leading coefficient of the polynomial $F$ can be sped up using directly Thomae's formula (cf. Theorem 11.1 in Gua02]). Second, the polynomial $F(X)$ is determined up to a fourth root of unity in $K$ (not only a sign, as stated in GJGG02]): the explicit determination of this unit is performed anyway as in loc. cit.

\section{Example: $A_{f}$ With ONLY ONE PRINCIPAL POlarization}

Let us consider the abelian surface $A_{f}$ attached to the normalized newform in $S_{2}\left(\Gamma_{0}(243)\right)$ :

$f=q+\sqrt{6} q^{2}+4 q^{4}-\sqrt{6} q^{5}+2 q^{7}+2 \sqrt{6} q^{8}-6 q^{10}+\sqrt{6} q^{11}-q^{13}+2 \sqrt{6} q^{14}+4 q^{16}+\cdots$.

We take the basis of regular differentials over $\mathbb{Q}$ given by

$$
\omega_{1}=\frac{1}{2}\left(f+{ }^{\sigma} f\right) \frac{d q}{q}, \quad \omega_{2}=\frac{1}{2 \sqrt{6}}\left(f-{ }^{\sigma} f\right) \frac{d q}{q} .
$$

The polarization $\mathcal{L}$ on $A_{f}$ induced by the canonical polarization on the Jacobian of $X_{0}(243)$ has type $[1,2]$. The period matrix of $A_{f}$ with respect to a symplectic basis $\left\{c_{1}, c_{2}, c_{3}, c_{4}\right\}$ of $H_{1}\left(A_{f}, \mathbb{Z}\right)$ and $\omega_{1}, \omega_{2}$ is $\Omega=\left(\Omega_{1} \mid \Omega_{2}\right)$ with

$$
\begin{gathered}
\Omega_{1}=\left(\begin{array}{cc}
12.3724 \cdots+21.4297 \ldots I & 13.5178 \cdots+7.8045 \ldots I \\
-2.253 \cdots+3.9023 \ldots I & -12.3724 \cdots+7.1432 \ldots I
\end{array}\right), \\
\Omega_{2}=\left(\begin{array}{cc}
-12.3724 \cdots+21.4297 \ldots I & -15.609 \ldots I \\
2.253 \cdots+3.9023 \ldots I & -14.2865 \ldots I
\end{array}\right),
\end{gathered}
$$

where $I=\sqrt{-1}$. Over $K=\mathbb{Q}(\sqrt{-3})$ the endomorphism algebra of $A_{f}$ is $\operatorname{End}_{K}^{0}\left(A_{f}\right)$ $=\left(\frac{6,-3}{\mathbb{Q}}\right)=\mathbb{Q}(i, j)$, with $i^{2}=6, j^{2}=-3$. Applying formula (3) we determine the matrices giving the action of the quaternions $i, j$ on $H_{1}\left(A_{f}, \mathbb{Z}\right)$. We find:

$$
i:\left(\begin{array}{rrrr}
0 & -2 & 0 & -2 \\
-1 & 0 & 1 & 0 \\
0 & 4 & 0 & -2 \\
-2 & 0 & -1 & 0
\end{array}\right), \quad j:\left(\begin{array}{rrrr}
-1 & 0 & -2 & 0 \\
0 & -1 & 0 & 2 \\
2 & 0 & 1 & 0 \\
0 & -2 & 0 & 1
\end{array}\right) .
$$

The criterion given in Lemma 3.1 shows that $\frac{1}{2} i+\frac{1}{6} k, \frac{1}{2}+\frac{1}{2} j, \frac{1}{3} k$ are also endomorphisms of $A_{f}$. Since $\mathcal{O}=\left\langle 1, \frac{1}{2} i+\frac{1}{6} k, \frac{1}{2}+\frac{1}{2} j, \frac{1}{3} k\right\rangle$ is a maximal order in $\left(\frac{6,-3}{\mathbb{Q}}\right)$, it must be $\operatorname{End}_{K}\left(A_{f}\right)=\mathcal{O}$. Hence, by Theorem 2.2 there exists only one isomorphism class of principal polarizations on $A_{f}$; it is defined over $K$ but not over $\mathbb{Q}$ by Proposition 2.3. A principal polarization must be of the form $\mathcal{L}^{\left(\gamma^{-1}\right)}$ for a totally positive symmetric endomorphism $\gamma \in \mathcal{O}$ with reduced norm 2. Indeed, we take

$$
\gamma=2+\frac{1}{2} i-\frac{1}{6} k:\left(\begin{array}{rrrr}
2 & 0 & 0 & -2 \\
0 & 2 & 1 & 0 \\
0 & 2 & 2 & 0 \\
-1 & 0 & 0 & 2
\end{array}\right)
$$


The Riemann form attached to $\mathcal{L}^{\left(\gamma^{-1}\right)}$ is $E_{\gamma^{-1}}(x, y)=E\left(\gamma^{-1} x, y\right)$ where $E$ is the Riemann form attached to $\mathcal{L}$. Thus, in the basis $\left\{c_{1}, c_{2}, c_{3}, c_{4}\right\}$ of $H_{1}\left(A_{f}, \mathbb{Z}\right)$ it is given by the matrix

$$
E_{\gamma}:\left(\begin{array}{rrrr}
0 & 0 & 1 & 0 \\
0 & 0 & 0 & 2 \\
-1 & 0 & 0 & 0 \\
0 & -2 & 0 & 0
\end{array}\right) \gamma^{-1}=\left(\begin{array}{rrrr}
0 & -1 & 1 & 1 \\
1 & 0 & 1 & 2 \\
-1 & -1 & 0 & 0 \\
-1 & -2 & 0 & 0
\end{array}\right) .
$$

With respect to $E_{\gamma^{-1}}$, a symplectic basis is $\left(c_{1}^{\prime}, c_{2}^{\prime}, c_{3}^{\prime}, c_{4}^{\prime}\right)={ }^{t} M .\left(c_{1}, c_{2}, c_{3}, c_{4}\right)$, with

$$
M=\left(\begin{array}{rrrr}
0 & 1 & 1 & 0 \\
0 & -1 & 0 & 0 \\
-1 & 0 & 0 & 1 \\
0 & -1 & 0 & -1
\end{array}\right)
$$

so that a period matrix for $\left(A_{f}, \mathcal{L}^{\left(\gamma^{-1}\right)}\right)$ is $\Omega_{\gamma}:=\Omega . M$. We can finally apply the procedure described in GJGG02 to this new period matrix to find a hyperelliptic curve $C$ over $K$ with $(\operatorname{Jac}(C), \Theta) \stackrel{K}{\simeq}\left(A_{f}, \mathcal{L}^{\left(\gamma^{-1}\right)}\right)$, where $\Theta$ denotes the canonical principal polarization on $\operatorname{Jac}(C)$. We obtain $Y^{2}=F(X)$, where:

$$
\begin{aligned}
F(X)= & \frac{4(3-2 \sqrt{-3})}{9} X^{6}+\frac{8(-1+\sqrt{-3})}{3} X^{5}+\frac{4(3-7 \sqrt{-3})}{9} X^{4} \\
& +\frac{2(7+23 \sqrt{-3})}{27} X^{3}-\frac{(11+7 \sqrt{-3})}{18} X^{2}+\frac{(15+\sqrt{-3})}{108} X+\frac{(-2+\sqrt{-3})}{324} .
\end{aligned}
$$

As we know that there is only one isomorphism class of principal polarizations on $A_{f}$, by Torelli's theorem, the curves $C$ and $\bar{C}$ must be isomorphic. Thus, their common Igusa invariants must lie in $\mathbb{Q}$. Indeed, they are

$$
\left\{i_{1}, i_{2}, i_{3}\right\}=\left\{\frac{2^{18} \cdot 41^{5}}{3^{3}}, 2^{12} \cdot 3 \cdot 41^{3}, 2^{9} \cdot 7 \cdot 41^{2} \cdot 47\right\} .
$$

The curve $C$ has been obtained up to numerical approximations. Next, we shall prove that $\operatorname{Jac}(C)$ is $K$-isogenous to the abelian surface $A_{f}$. We point out that the procedure which we will use to determine the order $\operatorname{End}(\operatorname{Jac}(C))$ can be applied when $\operatorname{Jac}(C)$ has a unique class of principal polarizations and its endomorphism algebra has a quaternion of reduced norm equal to \pm 2 .

Proposition 4.1. Let $C: Y^{2}=F(X)$ be the genus two curve given above. Then, $\operatorname{Jac}(C)$ has multiplication by a maximal order of the quaternion algebra $\left(\frac{6,-3}{\mathbb{Q}}\right)$ and is $K$-isogenous to the abelian variety $A_{f}$.

Proof. We split the proof into five steps.

(i) $C / K$ does not admit a descent to $\mathbb{Q}$. It can be easily checked that $\operatorname{Aut}(C)=$ $\langle w\rangle$, where $w$ denotes the hyperelliptic involution. Let $\bar{C}: \bar{Y}=\bar{f}(\bar{X})$, where $\bar{f}$ is the complex conjugate of $f$. Then, the isomorphism $\varphi: C \longrightarrow \bar{C}$ given by

$$
(\bar{X}, \bar{Y})=\left(\frac{-1+\sqrt{-3}+(3-\sqrt{-3}) X}{2 \sqrt{-3}+6(1-\sqrt{-3}) X}, \frac{24 \sqrt{-3} Y}{(2 \sqrt{-3}+6(1-\sqrt{-3}) X)^{3}}\right)
$$

satisfies that $\bar{\varphi} \circ=w$. By Weil's criterion, this implies that $C$ does not admit a descent over $\mathbb{Q}$. 
(ii) $K$-linear relations between the entries of a period matrix of $\operatorname{Jac}(C)$. We will prove that a period matrix for the curve $C$ is

$$
\Omega=\left(\begin{array}{llll}
\alpha-\frac{1+\sqrt{-3}}{2} \alpha-(3-\sqrt{-3}) \beta & \frac{-1+\sqrt{-3}}{2} \alpha+\frac{3-\sqrt{-3}}{2} \beta & \frac{3+\sqrt{-3}}{2} \beta \\
\beta-\frac{3+\sqrt{-3}}{6} \alpha-\frac{1-\sqrt{-3}}{2} \beta & \frac{3+\sqrt{-3}}{12} \alpha-\frac{1+\sqrt{-3}}{2} \beta & \frac{3-\sqrt{-3}}{12} \alpha
\end{array}\right),
$$

where $(\alpha, \beta)=\int_{x_{3}}^{x_{1}}\left(\frac{d X}{Y}, \frac{X d X}{Y}\right)$.

First, we remark that a symplectic basis for $H_{1}(C, \mathbb{Z})$ is given by the paths $\gamma_{1}$, $\gamma_{2}, \gamma_{3}, \gamma_{4}$, enclosing, respectively, the segments $\left[x_{1}, x_{3}\right],\left[x_{4}, x_{5}\right],\left[x_{1}, x_{2}\right],\left[x_{5}, x_{6}\right]$, where

$$
\begin{array}{ll}
x_{1}=0.085 \cdots-0.130 \cdots I, & x_{2}=0.098 \cdots-0.181 \cdots I, \\
x_{3}=0.146 \cdots-0.232 \cdots I, & x_{4}=0.718 \cdots+0.253 \ldots I, \\
x_{5}=0.751 \cdots-0.187 \ldots I, & x_{6}=0.770 \cdots-0.018 \ldots I,
\end{array}
$$

are the roots of the polynomial $f(X)$ defining the curve $C$. It is well known how to build a period matrix for $C$. For instance, we can take

$$
\Omega=\left(\begin{array}{cccc}
\int_{x_{3}}^{x_{1}} \frac{d X}{Y} & \int_{x_{4}}^{x_{5}} \frac{d X}{Y} & \int_{x_{1}}^{x_{2}} \frac{d X}{Y} & \int_{x_{5}}^{x_{6}} \frac{d X}{Y} \\
\int_{x_{3}}^{x_{1}} \frac{X d X}{Y} & \int_{x_{4}}^{x_{5}} \frac{X d X}{Y} & \int_{x_{1}}^{x_{2}} \frac{X d X}{Y} & \int_{x_{5}}^{x_{6}} \frac{X d X}{Y}
\end{array}\right),
$$

where we integrate along the segments joining the different roots of $f(X)$, and the orientation of the paths and the determination of $Y=\sqrt{f(X)}$ is taken so that

$$
\Omega=\left(\begin{array}{cccc}
35.97 \ldots-7.80 \ldots I & -22.45 \ldots & -12.37 \ldots+21.43 \ldots I & 11.23 \ldots-7.80 \ldots I \\
3.36 \ldots-7.14 \ldots I & -15.73 \ldots & 2.25+3.90 \ldots I & 7.87 \ldots-7.14 \ldots I
\end{array}\right) .
$$

Three ingredients will be used to find relations between the entries of this matrix:

a) The isomorphism $\varphi: C \longrightarrow \bar{C}$ given in (4).

b) A Richelot isogeny: If $\operatorname{Jac}(C)$ is simple over $\overline{\mathbb{Q}}$, then for every subgroup $G$ of its 2 -torsion isomorphic to $(\mathbb{Z} / 2 \mathbb{Z})^{2}$, there is a genus two curve $C^{\prime}$ : $Y^{\prime 2}=G\left(X^{\prime}\right)$ and an isogeny $\phi: \operatorname{Jac}(C) \longrightarrow \operatorname{Jac}\left(C^{\prime}\right)$ whose kernel is $G$ and $\phi^{*}\left(X^{\prime i} d X^{\prime} / Y^{\prime}\right)=X^{i} d X / Y$ for $0 \leq i \leq 1$. It is called the Richelot isogeny attached to $G$ (cf. Section 3.1 in BoMe88) or Chapter 9 in CaFl96]).

c) We know (cf. p. 90 in Vig80) that in all maximal orders of an indefinite quaternion algebra every integer $d \in \mathbb{Z}$ is a reduced norm. In particular, if $\operatorname{End}(\operatorname{Jac}(C))$ is such an order, by taking $d=2$ there is an endomorphism of $\operatorname{Jac}(C)$ whose kernel $G$ is isomorphic to $(\mathbb{Z} / 2 \mathbb{Z})^{2}$. Therefore, if $\operatorname{Jac}(C)$ has a unique class of principal polarization, then $C$ must be isomorphic to the curve $C^{\prime}$ given by the Richelot isogeny attached to $G$.

In our case, to build a convenient Richelot isogeny, we take, following Bost and Mestre in BoMe88,

$$
(P, Q, R):=\left(\frac{4(3-2 \sqrt{-3})}{9}\left(X-x_{1}\right)\left(X-x_{3}\right),\left(X-x_{2}\right)\left(X-x_{6}\right),\left(X-x_{4}\right)\left(X-x_{5}\right)\right)
$$

and $\Delta$ equal to the determinant of the polynomials $P, Q, R$ with respect to the basis $1, X, X^{2}$, which turns out to be $\Delta=1 / 6$. We thus arrive at the genus two curve $C^{\prime}: Y^{\prime 2}=1 / \Delta U\left(X^{\prime}\right) V\left(X^{\prime}\right) W\left(X^{\prime}\right)$ where $U=[Q, R], V=[R, P]$ and $W=[P, Q]$, whose Jacobian is isogenous to Jac $C$. More precisely, $C^{\prime}$ is given by the equation 
$Y^{\prime 2}=G\left(X^{\prime}\right)$, with

$$
\begin{aligned}
& G\left(X^{\prime}\right)=\frac{4(2-\sqrt{-3})}{3} X^{\prime 6}+\frac{2(-9+7 \sqrt{-3})}{3} X^{\prime 5}+\frac{(2(5-9 \sqrt{-3})}{3} X^{\prime 4} \\
& +\frac{4(7+23 \sqrt{-3})}{27} X^{\prime 3}+\frac{4(-9-5 \sqrt{-3})}{27} X^{\prime 2}+\frac{8}{27} X^{\prime}+\frac{-3+2 \sqrt{-3}}{243}
\end{aligned}
$$

Let

$$
\begin{array}{ll}
x_{1}^{\prime}=0.040 \cdots-0.214 \cdots I, & x_{2}^{\prime}=0.112 \cdots-0.184 \cdots I, \\
x_{3}^{\prime}=0.149 \cdots-0.154 \cdots I, & x_{4}^{\prime}=0.606 \cdots-0.024 \cdots I, \\
x_{5}^{\prime}=0.808 \cdots+0.019 \cdots I, & x_{6}^{\prime}=1.069 \cdots-0.059 \cdots I,
\end{array}
$$

be the roots of the polynomial $G\left(X^{\prime}\right)$ defining the curve $C^{\prime}$. The description of the isogeny $\operatorname{Jac} C \rightarrow \operatorname{Jac} C^{\prime}$ in terms of the periods of $C$ and $C^{\prime}$ is given by the equalities $(i=0,1)$ :

$$
\begin{aligned}
\int_{x_{1}}^{x_{3}} \frac{X^{i} d X}{Y} & =2 \int_{x_{2}^{\prime}}^{x_{3}^{\prime}} \frac{X^{\prime i} d X^{\prime}}{Y^{\prime}}, & \int_{x_{1}}^{x_{2}} \frac{X^{i} d X}{Y} & =\int_{x_{3}^{\prime}}^{x_{1}^{\prime}} \frac{X^{\prime i} d X^{\prime}}{Y^{\prime}}, \\
\int_{x_{2}}^{x_{6}} \frac{X^{i} d X}{Y} & =2 \int_{x_{3}^{\prime}}^{x_{4}^{\prime}} \frac{X^{\prime i} d X^{\prime}}{Y^{\prime}}, & \int_{x_{3}}^{x_{5}} \frac{X^{i} d X}{Y} & =\int_{x_{2}^{\prime}}^{x_{5}} \frac{X^{\prime i} d X^{\prime}}{Y^{\prime}}, \\
\int_{x_{4}}^{x_{5}} \frac{X^{i} d X}{Y} & =2 \int_{x_{5}^{\prime}}^{x_{4}} \frac{X^{\prime i} d X^{\prime}}{Y^{\prime}}, & \int_{x_{5}}^{x_{6}} \frac{X^{i} d X}{Y} & =\int_{x_{4}^{\prime}}^{x_{6}} \frac{X^{\prime i} d X^{\prime}}{Y^{\prime}} .
\end{aligned}
$$

The trick to prove these equalities is the following: by construction, six of the integrals $\int_{x_{j}}^{x_{k}} \frac{X^{i} d X}{Y}$ on the left must be equal to either an integral $\int_{x_{j^{\prime}}^{\prime}}^{x_{k^{\prime}}^{\prime}} \frac{X^{\prime i} d X^{\prime}}{Y^{\prime}}$ or $2 \int_{x_{j^{\prime}}^{\prime}}^{x_{k^{\prime}}^{\prime}} \frac{X^{\prime i} d X^{\prime}}{Y^{\prime}}$ for some $j^{\prime}, k^{\prime}$; since these are all different, only a match is possible between the integrals on $C$ and the integrals on $C^{\prime}$; evaluating all the integrals to enough accuracy, we discard all but one possible equality, which must be true.

We can now relate the integrals $\int_{x_{j^{\prime}}^{\prime}}^{x_{k^{\prime}}^{\prime}} \frac{X^{\prime i} d X^{\prime}}{Y^{\prime}}$ with the periods of $C$ by using the isomorphism $\phi: C \longrightarrow C^{\prime}$ given by

$$
(X, Y) \mapsto\left(X^{\prime}, Y^{\prime}\right)=\left(\frac{1-\sqrt{-3}}{12 X}, \frac{\sqrt{-3} Y}{18 X^{3}}\right) .
$$

We have

$\phi\left(x_{1}\right)=x_{6}^{\prime}, \quad \phi\left(x_{2}\right)=x_{5}^{\prime}, \quad \phi\left(x_{3}\right)=x_{4}^{\prime}, \quad \phi\left(x_{4}\right)=x_{1}^{\prime}, \quad \phi\left(x_{5}\right)=x_{3}^{\prime}, \quad \phi\left(x_{6}\right)=x_{2}^{\prime}$,

and

$$
\phi^{*}\left(\frac{d X^{\prime}}{Y^{\prime}}\right)=\frac{3+\sqrt{-3}}{2} \frac{X d X}{Y}, \quad \phi^{*}\left(\frac{X^{\prime} d X^{\prime}}{Y^{\prime}}\right)=\frac{3-\sqrt{-3}}{12} \frac{d X}{Y} .
$$

From these relations we obtain, for instance

$$
\int_{x_{3}^{\prime}}^{x_{1}^{\prime}} \frac{d X^{\prime}}{Y^{\prime}}=\frac{3+\sqrt{-3}}{2} \int_{\phi^{-1}\left[x_{1}^{\prime}, x_{3}^{\prime}\right]} \frac{X d X}{Y} .
$$

The transformed path $\phi^{-1}\left[x_{3}^{\prime}, x_{1}^{\prime}\right]$ is drawn on the following graphic: 


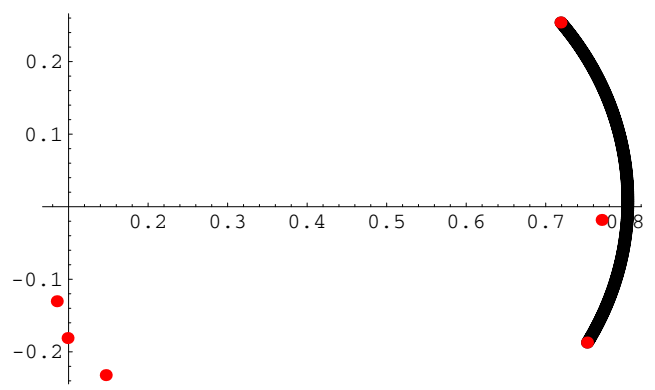

Hence, it is homologically equivalent to the path $\left[x_{4}, x_{6}\right]+\left[x_{6}, x_{5}\right]$, so that

$$
\int_{x_{1}^{\prime}}^{x_{3}^{\prime}} \frac{d X^{\prime}}{Y^{\prime}}=\frac{3+\sqrt{-3}}{2}\left(\int_{x_{4}}^{x_{6}}+\int_{x_{6}}^{x_{5}}\right) \frac{X d X}{Y} .
$$

Taking into account relations ([6), we obtain

$$
\int_{x_{1}}^{x_{2}} \frac{d X}{Y}=\frac{3+\sqrt{-3}}{2}\left(\int_{x_{4}}^{x_{6}}+\int_{x_{6}}^{x_{5}}\right) \frac{X d X}{Y} .
$$

In a similar way one can prove the following equalities:

$$
\begin{array}{lll}
\int_{x_{1}}^{x_{2}} \frac{d X}{Y}=\frac{3+\sqrt{-3}}{2}\left(\int_{x_{4}}^{x_{6}}+\int_{x_{6}}^{x_{5}}\right) \frac{X d X}{Y}, & \int_{x_{1}}^{x_{2}} \frac{X d X}{Y}=\frac{3-\sqrt{-3}}{12}\left(\int_{x_{4}}^{x_{6}}+\int_{x_{6}}^{x_{5}}\right) \frac{d X}{Y}, \\
\int_{x_{4}}^{x_{5}} \frac{d X}{Y}=-\frac{3+\sqrt{-3}}{2} \int_{x_{2}}^{x_{3}} \frac{X d X}{Y}, & \int_{x_{4}}^{x_{5}} \frac{X d X}{Y}=-\frac{3+\sqrt{-3}}{12} \int_{x_{2}}^{x_{3}} \frac{d X}{Y}, \\
\int_{x_{5}}^{x_{6}} \frac{d X}{Y}=-\frac{3+\sqrt{-3}}{2} \int_{x_{1}}^{x_{3}} \frac{X d X}{Y}, & \int_{x_{5}}^{x_{6}} \frac{X d X}{Y}=-\frac{3+\sqrt{-3}}{12} \int_{x_{1}}^{x_{3}} \frac{d X}{Y} .
\end{array}
$$

The last line gives immediately the expression of the last column of the period matrix $\Omega$ in terms of the first column. To relate the third column with the first and second columns of $\Omega$, we substitute the equalities $\int_{x_{2}}^{x_{3}}=\int_{x_{2}}^{x_{1}}-\int_{x_{1}}^{x_{3}}$ and $\int_{x_{4}}^{x_{6}}=$ $\int_{x_{4}}^{x_{5}}+\int_{x_{5}}^{x_{6}}$ in the first two equalities above, and look at the resulting equalities as a linear system of equations with respect to the integrals $\int_{x_{1}}^{x_{2}}$. Solving it, we find:

$$
\begin{aligned}
& \int_{x_{1}}^{x_{2}} \frac{d X}{Y}=\frac{3+\sqrt{-3}}{2} \int_{x_{4}}^{x_{5}} \frac{X d X}{Y}+\int_{x_{1}}^{x_{3}} \frac{X d X}{Y} \\
& \int_{x_{1}}^{x_{2}} \frac{X d X}{Y}=\frac{-3+\sqrt{-3}}{12} \int_{x_{4}}^{x_{5}} \frac{d X}{Y}+\int_{x_{1}}^{x_{3}} \frac{d X}{Y} .
\end{aligned}
$$

Hence, we have proved that

$$
\Omega=\left(\begin{array}{cccc}
\alpha & \delta & -\beta+\frac{3+\sqrt{-3}}{2} \gamma & \frac{3+\sqrt{-3}}{2} \beta \\
\beta & \gamma & -\alpha+\frac{-3+\sqrt{-3}}{12} \delta & \frac{3-\sqrt{-3}}{12} \alpha
\end{array}\right),
$$


for some $\alpha, \beta, \delta, \gamma \in \mathbb{C}$. Unfortunately, we do not obtain more information when we make a similar treatment from the intermediate equalities. At this point, the first ingredient of our proof plays its role, and the isomorphism $\varphi: C \rightarrow \bar{C}$. Note that

$$
\overline{\int_{x_{4}}^{x_{5}} \frac{X^{i} d X}{Y}}=\int_{\overline{x_{4}}}^{\overline{x_{5}}} \frac{\bar{X}^{i} d \bar{X}}{\bar{Y}},
$$

and we can pull back the last integrals to periods of $C$ by means of $\varphi$. The path $\varphi^{-1}\left[\overline{x_{4}}, \overline{x_{5}}\right]$ is homologous to $-\left[x_{1}, x_{2}\right]+\left[x_{2}, x_{3}\right]=-2\left[x_{1}, x_{2}\right]+\left[x_{1}, x_{3}\right]$, as seen in the following graphic:

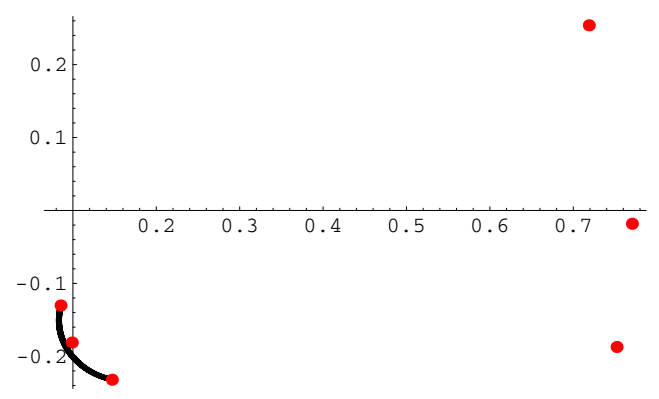

We obtain:

$$
\int_{\overline{x_{4}}}^{\overline{x_{5}}} \frac{\bar{X}^{i} d \bar{X}}{\bar{Y}}=\int_{\varphi^{-1}\left[\overline{x_{4}}, \overline{x_{5}}\right]} \varphi^{*} \frac{\bar{X}^{i} d \bar{X}}{\bar{Y}}=\left(-2 \int_{x_{1}}^{x_{2}}+\int_{x_{1}}^{x_{3}}\right) \varphi^{*} \frac{\bar{X}^{i} d \bar{X}}{\bar{Y}},
$$

where

$\varphi^{*} \frac{d \bar{X}}{\bar{Y}}=-\frac{1+\sqrt{-3}}{2} \frac{d X}{Y}+2 \sqrt{-3} \frac{X d X}{Y}, \quad \varphi^{*} \frac{\bar{X} d \bar{X}}{\bar{Y}}=\frac{-\sqrt{-3}}{3} \frac{d X}{Y}-\frac{1-\sqrt{-3}}{2} \frac{X d X}{Y}$.

Substituting these equalities in (8) and equating real and imaginary parts, we obtain four independent linear equations in the variables $\operatorname{Re}(\alpha), \operatorname{Im}(\alpha), \operatorname{Re}(\beta), \operatorname{Im}(\beta)$, $\operatorname{Re}(\delta), \operatorname{Im}(\delta), \operatorname{Re}(\gamma)$ and $\operatorname{Im}(\gamma)$. The solution of this system depending on the variables $\operatorname{Re}(\alpha), \operatorname{Im}(\alpha), \operatorname{Re}(\beta)$ and $\operatorname{Im}(\beta)$ yields the equality (5).

(iii) The ring $\operatorname{End}_{K}(\operatorname{Jac}(C))$ is a maximal order of $\left(\frac{6,-3}{\mathbb{Q}}\right)$. It can be checked now that the matrices

$$
T_{6}=\left(\begin{array}{ll}
0 & 1 \\
6 & 0
\end{array}\right), \quad T_{-3}=\left(\begin{array}{cc}
\sqrt{-3} & 0 \\
0 & -\sqrt{-3}
\end{array}\right)
$$

satisfy $T_{6}^{2}=6, T_{-3}^{2}=-3, T_{6} \cdot T_{-3}=-T_{-3} \cdot T_{6}$ and, by using (5), we have that

$$
\Omega \cdot\left(\begin{array}{rrrr}
-2 & -2 & 1 & 0 \\
-2 & 2 & -1 & -1 \\
-2 & 0 & 0 & -2 \\
-2 & 2 & -4 & 0
\end{array}\right)={ }^{t} T_{6} \cdot \Omega, \quad \Omega \cdot\left(\begin{array}{rrrr}
3 & -2 & -2 & 2 \\
2 & -3 & 0 & 2 \\
4 & 4 & -1 & 2 \\
0 & -4 & 2 & 1
\end{array}\right)={ }^{t} T_{-3} \cdot \Omega,
$$

which implies that there are $i, j \in \operatorname{End}_{K}(\operatorname{Jac}(C))$ such that the matrices with their actions on $H^{0}\left(C, \Omega^{1}\right)$ with respect to the basis $\{d X / Y, X d X / Y\}$ are $T_{6}$ and $T_{-3}$, respectively (see Lemma 3.1). Moreover, it is easy to check that the maximal order $\left\langle 1, \frac{1}{2} i+\frac{1}{6} k, \frac{1}{2}+\frac{1}{2} j, \frac{1}{3} k\right\rangle$ of $\mathbb{Q}(i, j)$ is contained in $\operatorname{End}_{K}(\operatorname{Jac}(C))$. To assert that this maximal order is the full ring endomorphisms of $\operatorname{Jac}(C)$, we only need to prove that $C$ is not isogenous to a square of an elliptic curve with complex multiplication; but, 
it follows from the fact that the reduction of $C$ at a prime of $K$ dividing to 7 (resp. 13 ) is isogenous to the square of an ordinary elliptic curve with multiplication by $\mathbb{Q}(\sqrt{-6})$ (resp. $\mathbb{Q}(\sqrt{-51})$ ).

(iv) $\operatorname{Jac}(C)$ is $K$-isomorphic to an abelian surface $A / \mathbb{Q}$ with $\operatorname{End}_{\mathbb{Q}}(A) \simeq \mathbb{Z}[\sqrt{6}]$. Let $u=1 / 2-j / 2+k / 3 \in \operatorname{Aut}(\operatorname{Jac}(C))$. Let $\iota$ be the isomorphism obtained as the composition of the following isomorphisms:

$$
\operatorname{Jac}(C) \stackrel{u}{\longrightarrow} \operatorname{Jac}(C) \stackrel{\varphi_{*}}{\longrightarrow} \mathrm{Jac}(\bar{C}),
$$

where $\varphi$ is as in (i). The matrix of the morphism

$$
\iota^{*}: H^{0}\left(\operatorname{Jac}(\bar{C}), \Omega_{\mathrm{Jac}(\bar{C}) / K}^{1}\right) \longrightarrow H^{0}\left(\operatorname{Jac}(C), \Omega_{\mathrm{Jac}(C) / K}^{1}\right)
$$

with respect the bases $\{d \bar{X} / \bar{Y}, \bar{X} d \bar{X} / \bar{Y}\}$ and $\{d X / Y, X d X / Y\}$ is the identity matrix. Therefore, $\iota \circ \iota=1$ and, then, by the Weil criterion there is an abelian surface $A / \mathbb{Q}$ and an isomorphism $\mu: \operatorname{Jac}(C) \longrightarrow A$ defined over $K$ such that $\bar{\mu}=\mu \circ \iota^{-1}$. Since $\nu=\mu \circ i \circ \mu^{-1}$ is invariant under the complex conjugation, it must be $\mathbb{Z}[\nu] \subseteq \operatorname{End}_{\mathbb{Q}}(A)$. Due to the fact that $\nu^{2}=6$ and $\mathbb{Z}[\sqrt{6}]$ is a maximal order, it follows that $\mathbb{Z}[\nu]=\operatorname{End}_{\mathbb{Q}}(A)$.

(v) $A$ is $\mathbb{Q}$-isogenous to $A_{f}$. The conductor of $\operatorname{Jac}(C)$ is the integer ideal of $K$ generated by $3^{8}$. By the recent progress with respect to the generalized ShimuraTaniyama-Weil Conjecture (cf. [KW06]), $A$ is modular and, thus, there exists a newform $g$ of level $M$ such that $A$ is $\mathbb{Q}$-isogenous to $A_{g}$. Since $g$ must have an inner-twist by the Dirichlet character $\chi$ attached to $K$, i.e. $g \otimes \chi={ }^{\sigma} g$, we have

$$
\operatorname{Res}_{K / \mathbb{Q}}(\operatorname{Jac}(C))=\operatorname{Res}_{K / \mathbb{Q}}(A) \stackrel{\mathbb{Q}}{\sim} A_{g} \times A_{g \otimes \chi}=A_{g}^{2} .
$$

By applying Milne's formula for the conductor of the Weil restriction (cf. Proposition 1 in [Mil72]) and the fact that cond $\left(A_{g}\right)=M^{2}$ (cf. [Car86]), we obtain that cond $\left(A_{g}\right)=3^{10}$. The assertion is obtained by checking that in the level $243=3^{5}$ the unique normalized newforms $g$ such that $A_{g}$ is a quaternionic surface are $f$ and its Galois conjugate.

Remark 4.1. As 2 divides the discriminant of the quaternion algebra $\operatorname{End}^{0}(\operatorname{Jac}(C))$, there is a unique ideal of norm 2 in any of its maximal orders. So, all quaternions of reduced norm 2, viewed as endomorphisms of $\operatorname{Jac}(C)$, have the same kernel and, thus, only one of the 15 curves obtained through a Richelot isogeny is isomorphic to $C$.

\section{Example: $A_{f}$ With no principal polarizations}

We can apply the same process to the normalized newform in $S_{2}\left(\Gamma_{0}(972)\right)$ :

$$
f=q+3 \sqrt{2} q^{5}+2 q^{7}+3 \sqrt{2} q^{11}-q^{13}-3 \sqrt{2} q^{17}+5 q^{19}+\cdots .
$$

The canonical polarization on $J_{0}(N)$ induces a polarization $\mathcal{L}$ on $A_{f}$ of type $[1,6]$. The endomorphism algebra of $A_{f}$ is $\operatorname{End}_{K}\left(A_{f}\right)=\left\langle 1, \frac{3}{2} i+\frac{1}{2} k, \frac{1}{2}+\frac{1}{2} j, k\right\rangle$, with $i^{2}=2, j^{2}=-3$. It has index 3 with respect to a maximal order in $\operatorname{End}_{K}^{0}\left(A_{f}\right)$. Since there are no symmetric totally positive elements of reduced norm 6 , we conclude that $A_{f}$ admits no principal polarizations. 


\section{Example: $A_{f}$ With TWo Principal POLARIZATIONS}

Let us finally consider the abelian surface $A_{f}$ attached to the normalized newform in $S_{2}\left(\Gamma_{0}(1568)\right)$,

$$
f=q+\sqrt{7} q^{3}-3 q^{5}+4 q^{9}-\sqrt{7} q^{11}-4 q^{13}-3 \sqrt{7} q^{15}+\cdots
$$

which corresponds to the entry labelled $S_{1568 L}$ in Table II. Now $i^{2}=7, j^{2}=-1$, and we find that $\operatorname{End}_{K}\left(A_{f}\right)=\mathbb{Z}\left\langle 1, \frac{1}{2}+\frac{1}{2} i+\frac{1}{2} j+\frac{1}{2} k, j, k\right\rangle$ is a maximal order in $\mathbb{Q}(i, j)$. The polarization $\mathcal{L}$ on $A_{f}$ induced by the canonical polarization on $J_{0}(1568)$ is of type $[1,14]$. By Theorem 2.2 there are two isomorphism classes of principal polarizations on $A_{f}$ over $K=\mathbb{Q}(\sqrt{-1})$, and by Lemma 2.3, they are not defined over $\mathbb{Q}$. Thus, there exist two $C_{1}, C_{2}$ over $K$ with $\operatorname{Jac}\left(C_{i}\right) \simeq A_{f}$. They correspond to the principal polarizations $\mathcal{L}^{\left(\gamma_{1}^{-1}\right)}$ and $\mathcal{L}^{\left(\gamma_{2}^{-1}\right)}$, where

$$
\gamma_{1}=7+2 i+k \quad \text { and } \quad \gamma_{2}=7-2 i+k
$$

A priori, both $C_{1}$ and $C_{2}$ could be $K$-isomorphic to their respective complex conjugates as above; if this is not the case, then they must be complex conjugates. Indeed, computations reveal that we are in this second case: the Igusa invariants of $C_{1}$ and $C_{2}$ are

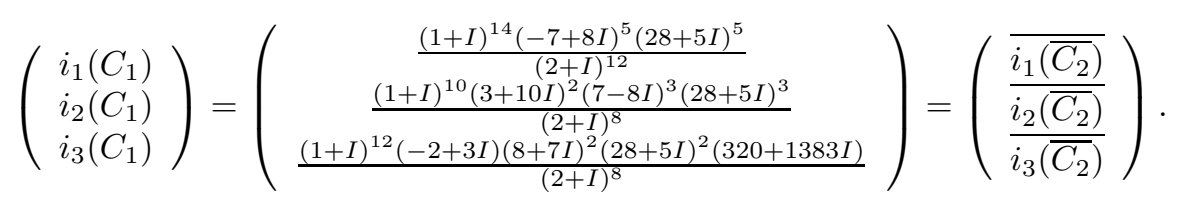

A rational equation for $C_{1}$ is

$$
\begin{aligned}
Y^{2}=\frac{1372-539 I}{5}( & X^{6}+\frac{332+208 I}{181} X^{5}+\frac{1173+2148 I}{1267} X^{4}+\frac{376+8060 I}{8869} X^{3} \\
& \left.-\frac{705-1992 I}{8869} X^{2}-\frac{1228-1612 I}{62083} X-\frac{607-492 I}{434581}\right) .
\end{aligned}
$$

It is worth mentioning that up to level $N \leq 2500$, there are only two modular abelian surfaces, $A_{f_{1}}$ and $A_{f_{2}}$, with quaternionic multiplication and more than one isomorphism class of principal polarizations. Both newforms have level $N=1568$ and, moreover, each of them is the twist of the other by the quadratic Dirichlet character of conductor 28 .

\section{TABle of QUATERnionic abelian SURFACES}

Using a program made by J. Quer for Magma we have found all quaternionic abelian surfaces $A_{f}$ which appear as subvariety of some $J_{0}(N)$ with $N \leq 7000$. In Table I below, we list for every level $N$ the number of factors $A_{f}$ with attached number fields $E, K$. The reduced discriminant of $\operatorname{End}^{0}\left(A_{f}\right)$ is denoted by $D$. 
For the $A_{f}$ in Table I with level $N \leq 2500$, we describe its endomorphism algebra and its principal polarizations in Table II. We follow the labelling of Magma to denote the $A_{f}$; for instance, $S_{243, D}$ denotes the fourth $\mathbb{Q}$-irreducible factor of $J_{0}(243)$. For every $A_{f}$ we describe the quadratic fields $E=\mathbb{Q}(\sqrt{m}), K=\mathbb{Q}(\sqrt{\delta})$, the endomorphism algebra $\operatorname{End}_{K}\left(A_{f}\right)$, its index $n$ with respect to a maximal order in $\operatorname{End}_{K}^{0}\left(A_{f}\right)$ and the numbers $\pi_{\mathbb{Q}}, \pi_{K}$ of polarizations over $\mathbb{Q}$ and $K$, respectively. For those $A_{f}$ admitting principal polarizations, we provide elements $\gamma$ originating them.

TABLE I

\begin{tabular}{|ccccc|ccccc|}
$N$ & $E$ & $K$ & $D$ & $\# A_{f}$ & $N$ & $E$ & $K$ & $D$ & $\# A_{f}$ \\
\hline \hline 243 & $\mathbb{Q}(\sqrt{6})$ & $\mathbb{Q}(\sqrt{-3})$ & 6 & 1 & 3136 & $\mathbb{Q}(\sqrt{7})$ & $\mathbb{Q}(\sqrt{-1})$ & 14 & 2 \\
675 & $\mathbb{Q}(\sqrt{2})$ & $\mathbb{Q}(\sqrt{-3})$ & 6 & 2 & 3886 & $\mathbb{Q}(\sqrt{6})$ & $\mathbb{Q}(\sqrt{-3})$ & 6 & 1 \\
972 & $\mathbb{Q}(\sqrt{2})$ & $\mathbb{Q}(\sqrt{-3})$ & 6 & 1 & 3886 & $\mathbb{Q}(\sqrt{2})$ & $\mathbb{Q}(\sqrt{-3})$ & 6 & 1 \\
1323 & $\mathbb{Q}(\sqrt{6})$ & $\mathbb{Q}(\sqrt{-3})$ & 6 & 2 & 3969 & $\mathbb{Q}(\sqrt{15})$ & $\mathbb{Q}(\sqrt{-7})$ & 15 & 1 \\
1568 & $\mathbb{Q}(\sqrt{7})$ & $\mathbb{Q}(\sqrt{-1})$ & 14 & 2 & 5184 & $\mathbb{Q}(\sqrt{3})$ & $\mathbb{Q}(\sqrt{-1})$ & 6 & 2 \\
1568 & $\mathbb{Q}(\sqrt{3})$ & $\mathbb{Q}(\sqrt{-1})$ & 6 & 2 & 5184 & $\mathbb{Q}(\sqrt{6})$ & $\mathbb{Q}(\sqrt{-3})$ & 6 & 2 \\
1849 & $\mathbb{Q}(\sqrt{6})$ & $\mathbb{Q}(\sqrt{-43})$ & 6 & 1 & 5292 & $\mathbb{Q}(\sqrt{10})$ & $\mathbb{Q}(\sqrt{-3})$ & 10 & 2 \\
2592 & $\mathbb{Q}(\sqrt{6})$ & $\mathbb{Q}(\sqrt{-1})$ & 6 & 2 & 5408 & $\mathbb{Q}(\sqrt{11})$ & $\mathbb{Q}(\sqrt{-1})$ & 22 & 2 \\
2592 & $\mathbb{Q}(\sqrt{3})$ & $\mathbb{Q}(\sqrt{-1})$ & 6 & 2 & 5408 & $\mathbb{Q}(\sqrt{3})$ & $\mathbb{Q}(\sqrt{-13})$ & 6 & 2 \\
2601 & $\mathbb{Q}(\sqrt{2})$ & $\mathbb{Q}(\sqrt{-51})$ & 6 & 1 & 6075 & $\mathbb{Q}(\sqrt{6})$ & $\mathbb{Q}(\sqrt{-3})$ & 6 & 2 \\
2700 & $\mathbb{Q}(\sqrt{10})$ & $\mathbb{Q}(\sqrt{-3})$ & 10 & 2 & 6400 & $\mathbb{Q}(\sqrt{6})$ & $\mathbb{Q}(\sqrt{-1})$ & 6 & 4 \\
3136 & $\mathbb{Q}(\sqrt{3})$ & $\mathbb{Q}(\sqrt{-1})$ & 6 & 2 & & & & & \\
\hline
\end{tabular}

TABLE II

\begin{tabular}{cccccccc}
$A_{f}$ & $(m, \delta)$ & $d$ & $\operatorname{End}_{K}\left(A_{f}\right)$ & $n$ & $\pi_{\mathbb{Q}}$ & $\pi_{K}$ & $\gamma$ \\
\hline \hline$S_{243 D}$ & $(6,-3)$ & 2 & $\left\langle 1, \frac{1}{2} i+\frac{1}{6} k, \frac{1}{2}+\frac{1}{2} j, \frac{1}{3} k\right\rangle$ & 1 & 0 & 1 & $2+\frac{1}{2} i-\frac{1}{6} k$ \\
\hline$S_{675 L}$ & $(2,-3)$ & 2 & $\left\langle 1, \frac{1}{2} i+\frac{1}{2} k, \frac{1}{2}+\frac{1}{2} j, k\right\rangle$ & 1 & 1 & 1 & $2+i$ \\
\hline$S_{675 P}$ & $(2,-3)$ & 2 & $\left\langle 1, \frac{1}{2} i+\frac{1}{2} k, \frac{1}{2}+\frac{1}{2} j, k\right\rangle$ & 1 & 1 & 1 & $2+i$ \\
\hline$S_{972 E}$ & $(2,-3)$ & 6 & $\left\langle 1, \frac{3}{2} i+\frac{1}{2} k, \frac{1}{2}+\frac{1}{2} j, k\right\rangle$ & 3 & 0 & 0 & \\
\hline$S_{1323 U}$ & $(6,-3)$ & 6 & $\left\langle 1, \frac{1}{2} i+\frac{1}{2} k, \frac{1}{2}+\frac{1}{2} j, k\right\rangle$ & 3 & 0 & 0 & \\
\hline$S_{1323 V}$ & $(6,-3)$ & 6 & $\left\langle 1, \frac{1}{2} i+\frac{1}{2} k, \frac{1}{2}+\frac{1}{2} j, k\right\rangle$ & 3 & 0 & 0 & \\
\hline$S_{1568 L}$ & $(7,-1)$ & 14 & $\left\langle 1, \frac{1}{2}+\frac{1}{2} i+\frac{1}{2} j+\frac{1}{2} k, j, k\right\rangle$ & 1 & 0 & 2 & $7+2 i+k$ \\
\hline$S_{1568 N}$ & $(7,-1)$ & 14 & $\left\langle 1, \frac{1}{2}+\frac{1}{2} i+\frac{1}{2} j+\frac{1}{2} k, j, k\right\rangle$ & 1 & 0 & 2 & $7+2 i+k$ \\
\hline$S_{1568 S}$ & $(3,-1)$ & 6 & $\left\langle 1, \frac{1}{2}+\frac{1}{2} i+\frac{1}{2} j+\frac{1}{2} k, j, k\right\rangle$ & 1 & 0 & 1 & $3+k$ \\
\hline$S_{1568 U}$ & $(3,-1)$ & 6 & $\left\langle 1, \frac{1}{2}+\frac{1}{2} i+\frac{1}{2} j+\frac{1}{2} k, j, k\right\rangle$ & 1 & 0 & 1 & $3+k$ \\
\hline$S_{1849 E}$ & $(6,-43)$ & 258 & $\left\langle 1, \frac{1}{2} i+\frac{1}{2} k, \frac{1}{2}+\frac{1}{2} j, k\right\rangle$ & 43 & 0 & 0 & \\
\hline
\end{tabular}




\section{REFERENCES}

[BCP97] W. Bosma, J. Cannon, and C. Playoust, The Magma algebra system. I. The user language, J. Symbolic Comput. 24 (1997), no. 3-4, 235-265.

[BoMe88] J-B. Bost and J-F. Mestre, Moyenne arithmético-géométrique et périodes des courbes de genre 1 et 2, Gaz. Math. 38 (1988), 36-64. MR970659 (89k:14072)

[BFGR06] N. Bruin, V. Flynn, J. González, and V. Rotger, On finiteness conjectures for modular quaternion algebras, Math. Proc. Camb. Philos. Soc., 141 (2006), no. 3,409-468.

[Car86] H. Carayol, Sur les représentations l-adiques associées aux formes modulaires de Hilbert, Ann. Sci. École Norm. Sup. (4), 19(1986), no. 3,383-408. MR870690 (89c:11083)

[CaFl96] J. W. S. Cassels and E. V. Flynn, Prolegomena to a middlebrow arithmetic of curves of genus 2, London Mathematical Society Lecture Note Series, 230, Cambridge University Press, (1996). MR1406090 (97i:11071)

[GGR05] J. González, J. Guàrdia, and V. Rotger, Abelian surfaces of $\mathrm{GL}_{2}$-type as Jacobians of curves, Acta Arith. 116 (2005), no. 3, 263-287.

[GJGG02] E. González-Jiménez, J. González, and J. Guàrdia, Computations on modular Jacobian surfaces, Algorithmic number theory (Sydney, 2002), Lecture Notes in Comput. Sci., vol. 2369, Springer, Berlin, 2002, pp. 189-197.

[Gua02] J. Guàrdia, Jacobian nullwerte and algebraic equations, Journal of Algebra 253 (2002), 112-132. MR1925010|(2004a:14032)

[KW06] C. Khare and J-P. Winterberger, Serre's modularity conjecture (I), preprint (2006). MR2254626 (2007e:11060)

[Mil72] J. S. Milne, On the arithmetic of abelian varieties, Invent. Math., 17 (1972), 177-190. MR0330174(48:8512)

[Rib80] K. A. Ribet, Twists of modular forms and endomorphisms of abelian varieties, Math. Ann. 253 (1980), no. 1, 43-62.

[Rot03] V. Rotger, Quaternions, polarization and class numbers, J. Reine Angew. Math. 561 (2003), 177-197.

[Shi71] G. Shimura, Introduction to the arithmetic theory of automorphic functions, Publications of the Mathematical Society of Japan, No. 11. Iwanami Shoten, Publishers, Tokyo, 1971, Kanô Memorial Lectures, No. 1.

[Shi73] G. Shimura, On the factors of the jacobian variety of a modular function field, J. Math. Soc. Japan 25 (1973), 523-544.

[Vig80] M.-F. Vignéras, Arithmétique des algèbres de quaternions, Lecture Notes in Mathematics 800, Springer (1980). MR580949 (82i:12016)

[Wei57] A. Weil, Zum Beweis des Torellischen Satzes, Nachr. Akad. Wiss. Göttingen. Math.Phys. Kl. IIa., (1957), 33-53. MR0089483 (19:683e)

Escola Politècnica Superior d'Engenyeria de Vilanova i la Geltrú, Avda Victor Balaguer S/N, 08800 Vilanova i la Geltrú, Spain

E-mail address: josepg@ma4.upc.edu

Escola Politècnica Superior d’Engenyeria de Vilanova i la Geltrú, Avda Victor Balaguer S/n, 08800 Vilanova i la Geltrú, Spain

E-mail address: guardia@ma4.upc.edu 